\title{
Evaluación de actitudes hacia los prejuicios sexuales (Machismo, doble moral y agresión sexual) en estudiantes universitarios de El Salvador
}

Por: Juan Carlos Sierra • José Ricardo Gutiérrez Quintanilla • Carlos Jesús Delgado Dominguez - Macarena de los Santos-Roig

\section{Introducción}

La presente investigación es un Proyecto de Cooperación conjunta entre la Universidad de Granada (España) y la Universidad Tecnológica de El Salvador, financiado por la
Conserjería de la Presidencia de Andalucía (España). El objetivo general fue evaluar los prejuicios sexuales y las actitudes sexuales negativas relacionadas con la violencia de género en población universitaria. La investigación estuvo conformado por dos estudios, el primero; formado por 506 estudiantes el cual pretendía analizar la fiabilidad y validez de los instrumentos; el segundo, formando por 1,499 estudiantes de las 6 universidades privadas más importantes del pais.

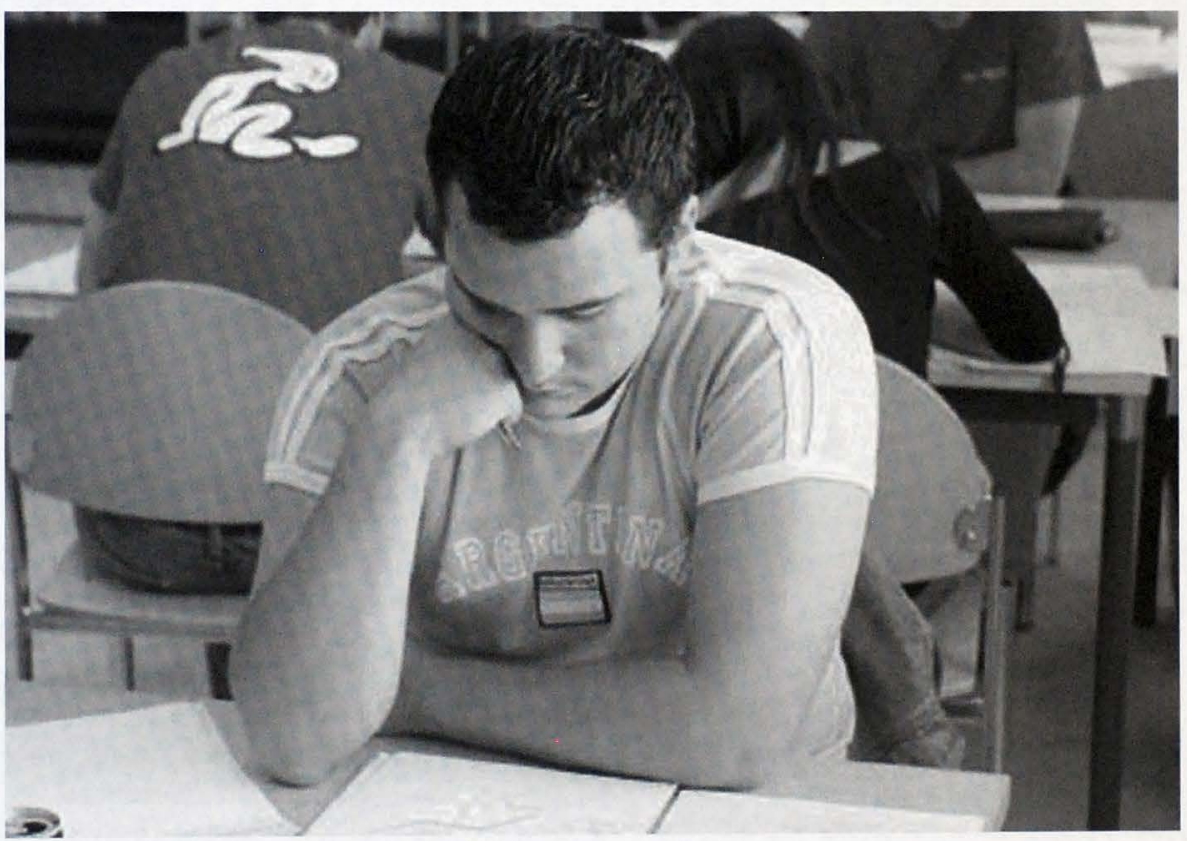


Evaluación de actitudes hacia los prejuicios sexuales

(Machismo, doble moral y agresión sexual)

en estudiantes universitarios de El Salvador pp. $53-58$

Sierra, J. C.

Gutiérrez Quintanilla, J. R., [et al]

Universidad Tecnológica de El Salvador - Agosto 2007 - №. 38

Los diseños de los estudios son no experimentales de tipo incidental, para el desarrollo del primer estudio (instrumental, León y Montero 2005) se aplico las normas internacionales para la construcción/adaptación de instrumentos y aparatos. El primero se realizo con estudiantes de la asignatura de psicologia general de la Universidad Tecnológica de El Salvador (UTEC) siendo esta una asignatura que la cursan la mayoria de carreras que se sirven en la UTEC. Tras los análisis estadísticos de fiabilidad y validez de los 9 instrumentos se seleccionaron 8 de estos con los cambios y ajustes pertinentes. Después de haber depurado los instrumentos, se envío una carta de solicitud a los rectores de 8 universidades solicitándoles autorización para administrar una bateria de pruebas, de estas aceptaron participar; La Universidad Don Bosco (UDB), Universidad Centroamericana(UCA), Universidad Evangélica de EI Salvador (UEES), Universidad Modular Abierta(UMA), la Universidad Francisco Gavidia(UFG) y Universidad Tecnológica de EI Salvador (UTEC), no colaboraron la Universidad de El Salvador (UES) y la Universidad Dr. José Matias Delgado(UJMD).

\section{Método}

Participantes.

Este informe está centrado en los resultados del $2^{\circ}$ estudio, la muestra seleccionada mediante muestreo incidental estuvo formada por 1.499 estudiantes universitarios de los que $699(46,6 \%)$ fueron hombres y 800 $(53,4 \%)$ mujeres. El rango de edad osciló entre los 18 y 40 años tanto para hombres $(M=22,39$ años; $D T=$ 4,63 ) como para mujeres $(M=21,89$ años; $D T=4,10$ ). Todos ellos son estudiantes universitarios pertenecientes a distintas universidades de San Salvador (EI Salvador): Universidad Tecnológica de El Salvador (UTEC) (22,8\%), Universidad Francisco Gavidia (UFG) $(20,4 \%)$, Universidad Centroamericana José Simeón Cañas (UCA) (19\%), Universidad Modular Abierta (UMA) $(14,1 \%)$, Universidad Don Bosco (UDB) $(12,1 \%)$ y la Universidad Evangélica (UEV) $(11,5 \%)$. En relación al tipo de estudios que cursaban, la mayor parte de los estudiantes fueron de Ingeniería en Sistemas y Computación (17,8\%), se distribuyeron de la siguiente forma: Ciencias Jurídicas (15,6\%), Administración de Empresas $(10,7 \%)$, Ingeniería Industrial $(9 \%)$, Idiomas (6,5\%), Mercadotecnia $(5,8 \%)$, Relaciones Públicas y Comunicación $(5,4 \%)$, Psicología $(4,8 \%)$, Ciencias de la Educación $(4,6 \%)$, Contaduría Pública $(4,3 \%)$, Medicina (3,8\%), Estudios Técnicos $(3,6 \%)$, Ingenieria de Telecomunicaciones $(1,7 \%)$, Informática $(1,2 \%)$, Ingeniería Civil $(1,1 \%)$, Ingenieria Mecánica (1\%), Ingenieria Eléctrica $(0,8 \%)$, Lengua y Literatura $(0,8 \%)$, Ingeniería Química $(0,6 \%)$,
Economía $(0,6 \%)$ e Ingeniería de Automatización (0,3\%).

La mayor parte de la muestra estuvo compuesta por estudiantes de primer año de carrera $(38,3 \%)$; un $23,2 \%$ fueron de segundo año, un $17,1 \%$ de tercer año, un $12,3 \%$ de cuarto y un $8,9 \%$ de quinto año. Un $30,8 \%$ vivia en una población de menos de 15.000 habitantes, un $35,4 \%$ residia en poblaciones de entre 15.000 - 50.000 habitantes $y$ el $33,8 \%$ en una población de más de 50.000 habitantes. La mayor parte de los estudiantes vivía con sus padres $(74,2 \%)$, un $10,5 \%$ vivia con su pareja, un $9,2 \%$ con parientes y el resto bien vivia sólo $(4,6 \%)$ o bien con amigos $(1,4 \%)$. En un $28,9 \%$ de la muestra, los padres tenían un nivel de estudios básicos, un $31 \%$ tenia el $\mathrm{Ba}$ chillerato, un $34,3 \%$ tenia estudios universitarios y el resto tenía estudios técnicos (1,8\%) o no tenía estudios $(1,1 \%)$. El $33,9 \%$ de las madres tenía estudios básicos, un $37,7 \%$ tenía el Bachillerato, un $22,9 \%$ estudios universitarios, un $2,3 \%$ estudios técnicos y, por último, un $1,7 \%$ no tenía estudios. Respecto a la ocupación el $56,1 \%$ de los padres trabajaban en el sector de Administración y Servicios, el $26,4 \%$ pertenecia al ámbito profesional (licenciados, ingenieros y maestros), el $6 \%$ trabajaba en el sector Agrícola y Ganadero, un 5,7\% estaba jubilado, el $4,2 \%$ pertenecía al sector 
empresarial, el $0,8 \%$ eran del sector sanitario y por último el $0,8 \%$ de los padres estaban desempleados. En cuanto a la ocupación de la madre, el $74,9 \%$ pertenecía al sector de Administración y Servicios, el $16,8 \%$ eran profesionales, el $3,1 \%$ trabajaba en el ámbito sanitario, el $2,5 \%$ pertenecia al sector empresarial, un $2 \%$ estaba jubilada, un $0,6 \%$ desempleada y un $0,1 \%$ trabajaba en el sector Agricola y Ganadero. Poco más de la mitad de los estudiantes evaluados (51\%) había tenido pareja estable desde hacia tres meses, un $97,3 \%$ se consideraba de orientación heterosexual, un $41,7 \%$ tenía una práctica religiosa semanal y un $39,1 \%$ no tenía una orientación política definida.

\section{Instrumentos.}

Cuestionario sociodemográfico de elaboración propia, Escala de Deseabilidad Social (Social Desirability Scale, SDS) (Marlowe y Crowne, 1960); Escala de Doble Moral (Double Standard Scale, DSS) (Caron et al., 1993); Encuesta de Opinión Sexual (Sexual Opinion Survey, SOS) (Fisher et al., 1988); Cuestionario de Agresión (Agressive Questionnaire, $A Q$ ) (Buss y Perry, 1992); Inventario de Expresión de Ira Estado-Rasgo (The State-Trait Anger Expresión Inventory-2, STAXI-2) (Spielberger, 1996); Escala de Actitudes hacia la Violación (Rape Supportive Attitude Scale, RSAS) (Lottes, 1991);
Inventario de Conducta Sexual Agresiva (Agressive Sexual Behavior Inventory, ASBI) (Mosher y Anderson, 1986).

\section{Procedimiento.}

En primer lugar, se pidió permiso a las instituciones en las que se quiso llevar a cabo el estudio. De ocho universidades consultadas sólo respondieron seis. La administración de los instrumentos se llevó a cabo por dos investigadores en las diferentes aulas de las universidades en las que se consiguió el permiso. Durante el proceso de consentimiento informado verbal, se les indicó a los participantes que se trataba de un estudio cuyo objetivo era evaluar diversos aspectos de la sexualidad y que se estaba llevando a cabo en diferentes universidades de San Salvador. Se les aseguró que sus respuestas serian totalmente anónimas y que serian tratadas de modo confidencial. A pesar de no recibir ninguna gratificación por su colaboración, el $100 \%$ de los participantes colaboró de manera voluntaria.

\section{Algunos resultados}

Análisis estadisticos

Con el objetivo de determinar la influencia de diferentes variables sociodemográficas sobre las actitudes favorables hacia la violación, se empleó ANOVAy una prueba $t$ dependiendo de la variable utilizada; en el caso del $A S B I$ y aquellas variables relacionadas con el RSAS que no cumplian el supuesto de homogeneidad de varianzas, se utilizó la prueba no paramétrica $H$ de Kruskal-Wallis para varias muestras independientes. Por último, se estimó un modelo explicativo de las actitudes favorables hacia la violación y de la conducta sexual agresiva a través de diversas variables relacionadas con dichos constructos.

Influencia de variables sociodemográficas sobre las actitudes favorables hacia la violación.

Con el objetivo de conocer la posible influencia de diferentes variables sociodemográficas sobre las actitudes favorables hacia la violación se compararon en esta variable los diferentes grupos que se conformaron a partir de aquellas. Se presenta las puntuaciones medias $y$ desviaciones típicas que los hombres obtuvieron en la Escala de Actitudes Favorables hacia la Violación en función de diversas variables sociodemográ-ficas: universidad, carrera, año de carrera, orientación sexual, tamaño de la población de residencia, convivencia, estudios del padre, estudios de la madre, ocupación del padre, ocupación de la madre, práctica religiosa y orientación política. En el caso de los hombres, se encontró una correlación negativa 
entre actitudes favorables hacia la violación y edad $\left(r=-0,173^{\star \star} ; p<\right.$ $0,01)$; aunque la correlación es más bien modesta deja entrever que con la edad las actitudes hostiles hacia la mujer tienden a disminuir. En el resto de variables, el ANOVA no mostró diferencias significativas entre los distintos grupos. Por lo que respecta a la variable pareja se llevó a cabo una prueba $t$ para muestras independientes, obteniéndose una puntuación media menor en actitudes favorables hacia la violación en los participantes con pareja $\left(t_{299}=-2,940 ; p<0,004\right)$.

En el caso de las mujeres, se encontraron diferencias significativas en actitudes favorables hacia la violación en función de algunas de las variables sociodemográficas: universidad, carrera, año de carrera, ocupación del padre y orientación política. En el caso de las dos primeras variables, al no cumplirse el supuesto de homogeneidad de varianzas, se utilizó la prueba no paramétrica $H$ de Kruskal-Wallis para varias muestras independientes. Dicha prueba arrojó diferencias significativas, tanto en la variable universidad $\left(X^{2}=15,57 ; p\right.$ $<0,008)$ como carrera $\left(X^{2}=32,06\right.$; $p<0,015)$. En el resto de variables, el ANOVA, indicó diferencias significativas en año de carrera $F_{(5,}$ $\left.{ }_{655)}=6,43 ; p=0,000\right)$, ocupación del padre $F_{(6,565)}=2,49 ; p=0,022, y$ orientación política $F_{(3,674)}=6,39 ; p$ $=0,000$.
Las comparaciones realizadas a posteriori mediante la prueba de Scheffé indicaron en relación al año de carrera, que los sujetos de primer año tienen más actitudes favorables hacia la violación que los de quinto año $(p=0,00)$. En cuanto a la ocupación del padre las pruebas a posteriori no arrojaron diferencias significativas entre grupos. En relación a la orientación política, los que decian no tener ninguna orientación mostraron puntuaciones más altas que los que se consideraban de centro $(p=$ $0,00)$ Para las variables universidad y carrera se utilizó la prueba de $U$ Mann Whitney. En relación a las universidades, hubo diferencias significativas entre la Universidad Don Bosco y la Universidad Francisco Gavidia $(Z=-2,43 ; p=$ $0,015)$; entre la Universidad Don Bosco y la Universidad Modular Abierta $(Z=-3,03 ; p=0,02)$; entre la Universidad Francisco Gavidia y la Universidad Centroamericana "José Simeón Cañas" ( $Z=-1,98 ; p$ $=0,048) ; y$, por último, entre la Universidad Modular Abierta y las universidades Centroamericana "José Simeón Cañas" $(Z=-2,94 ; p$ $=0,003)$, Evangélica $(Z=-2,44 ; p$ $=0,015)$ y Tecnológica $(Z=-1,98$; $p=0,047$ ). Se pueden apreciar las diferencias de medias en actitudes favorables hacia la violación en función de la variable carrera.

Con el objetivo de conocer la influencia que determinadas

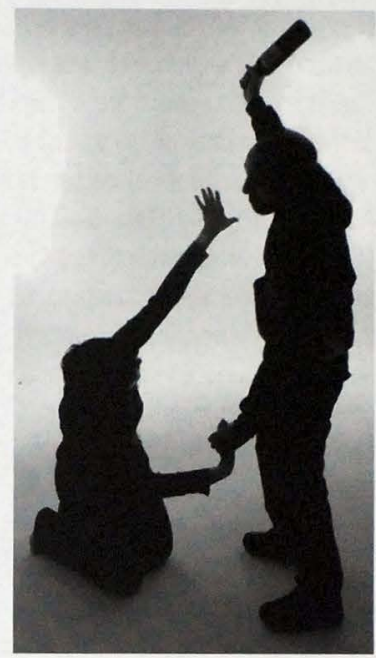

variables sociodemográficas pudieran tener sobre la conducta sexual agresiva, se compararon en esta variable los diferentes grupos que se conformaron a partir de aquellas. Se presentan las puntuaciones medias y las desviaciones típicas obtenidas por los hombres en el $A S B I$ en función de las distintas variables sociodemográficas: universidad, carrera, año de carrera, tener pareja, orientación sexual, tamaño de la población de residencia, convivencia, estudios del padre, estudios de la madre, ocupación del padre, ocupación de la madre, práctica religiosa y orientación política. Para ello se usó la prueba no paramétrica $H$ de KruskallWallis para varias muestras independientes. Dicha prueba arrojó diferencias significativas, en las variables carrera $\left(X^{2}=31,52\right.$; 
$p<0,049)$, año de carrera $\left(X^{2}=\right.$ $12,99 ; p<0,011)$ y práctica religiosa $\left(X^{2}=10,26 ; p<0,036\right.$. Por lo que respecta a la variable pareja se llevó a cabo una prueba $t$ para muestras independientes, no obteniéndose diferencias significativas entre los hombres con pareja y sin pareja $\left(t_{471}=1,13\right.$; $p<0,259)$. La edad no correlacionó de forma significativa con la conducta sexual agresiva ( $r$ $=0,04 p=0,38$ ).

Las comparaciones realizadas a posteriori mediante la prueba de $U$ Mann Whitney indicaron en relación al año de carrera, diferencias significativas entre el primer y el cuarto $(Z=-3,03 ; p=0,002)$ y quinto año $(Z=-2,73 ; p=0,018)$; entre el segundo y cuarto año $(Z=-2,41 ; p$ $=0,016) ; y$, por último, entre el tercer y cuarto año de carrera $(Z=$ $-2,22 ; p=0,026)$. En relación a la práctica religiosa, hay diferencias significativas entre la práctica diaria y la práctica mensual $(Z=-2,53 ; p$ $=0,011)$, alguna vez al año $(Z=$ $2,02 ; p=0,042)$ y sin práctica religiosa $(Z=-2,30 ; p=0,021)$; también se encontraron diferencias entre la práctica semanal y mensual $(Z=-1,97 ; p=0,049)$. Se pueden apreciar las diferencias de medias en conducta sexual agresiva en función de las variables año de carrera y práctica religiosa. Para una información más detallada de las puntuaciones medias en el ASBl en función de la variable carrera.

\section{Discusión}

Entre los resultados que se obtuvieron están: Correlaciones significativas entre la doble moral sexual y la ideología política; concretamente, aquellos que se consideraban de una ideología política de centro-derecha puntuaron más alto en doble moral sexual frente a los que no se identificaban con ninguna o se consideraban con una ideología de izquierdas., con respecto a la influencia de las variables sociodemográficas en la conducta sexual agresiva de los hombres, se encontraron diferencias significativas en función de la carrera, año de carrera y práctica religiosa. En el grupo de hombres las variables que correlacionaron significativamente con las actitudes favorables hacia la violación fueron ira, hostilidad, agresión física, agresión verbal, erotofilia y doble moral; en la muestra de mujeres las variables asociadas con el constructo actitudes favorables hacia la violación fueron ira, hostilidad, agresión física, erotofobia y doble moral. Algunas conclusiones: Los prejuicios sexuales siguen estando presentes en la población universitaria, entre ellos se tienen: Doble moral sexual $30,61 \%$ en hombres y $15,42 \%$ en mujeres, Actitudes favorables hacia la violencia contra la mujer: $27,17 \%$ y $19,08 \%$ en mujeres y Conducta sexual agresiva hacia la mujer: $11,60 \%$ en hombres.

Por lo que respecta a la influencia de variables sociodemográficas en la conducta sexual agresiva de los hombres, se encontraron diferencias significativas en ésta en función de la carrera, año de carrera y práctica religiosa. En este caso, a diferencia de las actitudes favorables hacia la violación, resultó una relación inversa entre el año de carrera y la ejecución de conductas sexuales agresivas; es decir, los alumnos de los últimos cursos obtuvieron medias más altas en la conducta sexual agresiva, por lo que en este caso la educación no resultó ser un factor modulador de posibles agresiones sexuales. La práctica religiosa si resultó ser un factor protector para la realización de agresiones sexuales, puesto que los sujetos con una alta frecuencia de prácticas religiosas puntuaron más bajo en agresividad sexual. Sin embargo, otros

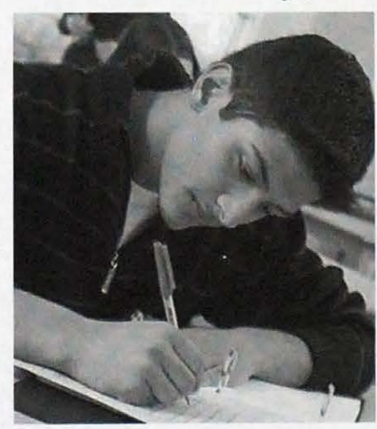


autores (Dieguez et al., 2003) han informado de la asociación entre la práctica religiosa y la doble moral. Estos datos son importantes teniendo en cuenta la elevada tasa de personas religiosas que posee Latinoamérica, por lo que respecta a este estudio, el $59 \%$ de los encuestados informó de una frecuencia de práctica religiosa diaria o semanal.

Por tanto, se puede decir que si se cumple la segunda de las hipótesis propuesta en este estudio, en la que se establecian diferencias en actitudes favorables hacia la violación y conducta sexual agresiva en función de variables sociodemográficas.

El último paso de este segundo estudio fue la construcción de un modelo explicativo capaz de predecir los dos constructos evaluados: las actitudes favorables hacia la violación y la conducta sexual agresiva. En este caso, se hipotetizó que las actitudes favorables hacia la violación se verán explicadas por una serie de variables relacionadas con el constructo. Para ello, el primer paso fue la búsqueda de variables que correlacionaran significativamente con dicho constructo. En el grupo de hombres las variables que correlacionaron significativamente con las actitudes favorables hacia la violación fueron ira, hostilidad, agresión física, agresión verbal, erotofilia y doble moral; en la muestra de mujeres las variables asociadas con el constructo actitudes favorables hacia la violación fueron ira, hostilidad, agresión física, erotofobia y doble moral. Dichas correlaciones se ven apoyadas por resultados similares encontrados en otros estudios (Murnen et al., 2002). Ortega et al. (2006) encontraron correlaciones entre una versión reducida de la RSAS y las variables ira, hostilidad, agresión verbal y agresión física. Tras esto se pasó a estimar un modelo de regresión de actitudes favorables hacia la violación tanto en hombres como en mujeres. En el caso de los hombres fueron las variables hostilidad, agresión física y doble moral la que explicaron el $22 \%$ de las actitudes favorables hacia la violación; en el caso de las mujeres las variables que explicaron el $22 \%$ de las actitudes favorables hacia la violación, fueron hostilidad y doble moral. Los resultados encontrados ponen de manifiesto que no todas las variables evaluadas influyen del mismo modo sobre las actitudes favorables hacia la violación, es decir unas tienen más peso que otras; y éste, a su vez, es parecido en hombres y mujeres, pues son las variables hostilidad y doble moral las que más varianza explican en cada caso. No obstante debemos añadir, que el porcentaje que explicaron este conjunto de variables fue moderado, lo cual indica que nos enfrentamos a un fenómeno complejo.

\section{Conclusiones}

- Las variables sociodemográficas (edad, tener pareja, Universidad, carrera, año de carrera, orientación política y práctica religiosa) se asocian a los prejuicios sexuales, pero cambian en función del género.

- Las actitudes favorables hacia la violación se explican básicamente por la doble moral sexual, tanto en hombres como en mujeres, aunque el porcentaje de varianza explicado es moderado $(22 \%)$.

- Múltiples factores explican la conducta sexual agresiva hacia las mujeres: ira, agresión verbal, actitudes sexuales negativas, doble moral sexual $y$ actitudes favorables hacia la violación, siendo el porcentaje final de varianza explicada moderado (18\%).

- Los prejuicios sexuales siguen estando presentes en la población universitaria.

- Doble moral sexual: $30,61 \%$ en hombres y $15,42 \%$ en mujeres. - Actitudes favorables hacia la violencia contra la mujer: $27,17 \%$ y $19,08 \%$ en mujeres

- Conducta sexual agresiva hacia la mujer: $11,60 \%$ en hombres. 